\title{
28 Research Square \\ Force Generation of the Hallux is More Sensitive to the Ankle and Metatarsophalangeal Joint Angle Than the Lesser Toes
}

Junya Saeki ( $\boldsymbol{\sim}$ j.saeki@kurenai.waseda.jp )

Waseda University https://orcid.org/0000-0002-9384-7475

Soichiro Iwanuma

Teikyo University of Science

Suguru Torii

Waseda University

\section{Research}

Keywords: toe flexor muscle strength, intrinsic foot muscle, extrinsic foot muscle, torque-angle relationships, toe grip strength

Posted Date: July 30th, 2020

DOl: https://doi.org/10.21203/rs.3.rs-49961/v1

License: (c) (1) This work is licensed under a Creative Commons Attribution 4.0 International License. Read Full License 


\section{Abstract}

Background: Because the structure of the hallux is independent of that of the lesser toes and it uses different muscles to move, the force generation characteristics of the hallux could be independent of those of the lesser toes. The purpose of this study is to clarify the torque-angle relationships in the first and second-fifth metatarsophalangeal joints (MTPJs).

Methods: Ten healthy young men served as volunteers in this study. The maximal voluntary contraction (MVC) of the plantar-flexion torques of the first and second-fifth MTPJs were measured at $0^{\circ}, 15^{\circ}, 30^{\circ}$, and $45^{\circ}$ dorsiflexed positions of the MTPJs and $20^{\circ}$ plantar-flexed, neutral, and $20^{\circ}$ dorsiflexed positions of the ankle. The Friedman test and the Wilcoxon signed-rank test with a Holm correction was used for the ankle and MTPJ angles.

Results: When the first MTPJ was $0^{\circ}$ to DF30 was higher than at $\mathrm{PF} 20^{\circ}$ of the ankle. On the other hand, no significant difference existed between the MVC torques of the second-fifth MTPJs at any ankle position. When the ankle was in a neutral position, the MVC of the first MTPJ torque increased as the MTPJ was dorsiflexed. However, the MVC torques of the second through fifth MTPJs did not significantly differ for the $15^{\circ}, 30^{\circ}$, and $45^{\circ}$ dorsiflexed positions of the MTPJ.

Conclusion: The MVC torque of the first MTPJ is more sensitive to the MTPJ and ankle positions than the second-fifth MTPJs.

\section{Background}

The toe flexor muscles are activated during walking and holding a single-leg stance [1, 2]. It has been reported that toe grip strength is associated with functional mobility in the older people [3]. Consequently, the weakness of the toe grip is a risk factor for falls in the older people [4]. In addition, in healthy young people, the cross-sectional area of the intrinsic foot muscles affects their performance during the singleleg stance [5]. Thus, the toe flexor muscle function affects the balance ability for all ages, and the assessment of the toe flexor muscle strength is important for the improvement of the balancing ability in humans.

The joint angle alters the maximum force generated by the muscle [6]. Additionally, it affects the muscle length and internal moment arm [7, 8], which further affect the force generation capacity. The length of the extrinsic toe flexor muscles is altered by the metatarsophalangeal joint (MTPJ) as well as the ankle joint angle [7]. The plantar flexion torque of the MTPJ is influenced by the force-length relationship of the intrinsic and extrinsic muscles of the foot when the MTPJ angle is changed, and it is influenced by the relationship with the extrinsic muscles when the ankle joint angle is changed. During the plantar-flexion of the ankle, the soleus muscle is activated selectively at the knee flexion position [9]. Measuring muscle strength at various joint angles helps us determine the measurement positions of the maximal voluntary contraction (MVC) torque according to the muscle that needs to be assessed. 
A previous study reported the relationship between the MVC torque and joint angle (torque-angle relationship) of an MTPJ for all toes [10]. However, the structure of the first toe (hallux) is independent of that of the other toes and it is moved using different muscles in most primates, including humans [11]. Our previous study reported that runners with a history of medial tibial stress syndrome exhibit higher plantar-flexion strength in their first MTPJ [12]. Regarding adaptation in sports, this study suggests that it is important to consider the strengths of the hallux and lesser toes separately. Moreover, the composition ratio of the intrinsic and extrinsic muscles is different between the plantar-flexion muscle of the first and second-fifth MTPJs [13]. Consequently, there is a possibility that the sensitivity of the plantar-flexion muscle to the joint angle is different in case of first and second-fifth MTPJs.

Recently, we developed a device that could measure the plantar-flexion torque of the first MTPJ and a unit of the second-fifth MTPJs [14]. It is possible to measure the plantar-flexion torque of the first MTPJ and second-fifth MTPJs at various MTPJ and ankle positions. The purpose of this study was to determine the relationship between the MVC of the plantar-flexion torque and joint angle in the first and secondfifth MTPJs using this device. We hypothesized that the plantar-flexion of the first MTPJ is sensitive to the joint positions due to the large composition ratio of the extrinsic muscles.

\section{Methods}

\section{Participants}

Ten healthy young men voluntarily participated in this study. The mean \pm standard deviations of their age, height, and mass were $23.2 \pm 1.9$ years, $169.2 \pm 3.7 \mathrm{~cm}$, and $58.4 \pm 8.1 \mathrm{~kg}$, respectively. The individual values are detailed in Table 1. The required sample size for a repeated analysis of variance [effect size $=$ 0.25 , a error $=0.05$, power $=0.80$, correlation among repeated measures $=0.6]$ was calculated using a statistical power analysis software (G* power 3.1, Heinrich Hein University, Germany), and the value obtained was 10 . This study was approved by institutional human research ethics committee and was carried out in accordance with the declaration of Helsinki.

\section{Procedures}

\section{Measurement of MVC torque}

The MVC torque was measured using a custom-made torque-measuring device. This device recorded the tensile force data from the strain gauge (TU-BR, TEAC, Japan), and converted it from analog to digital using an A/D converter (Power Lab, AD instruments, Australia) via an amplifier (DPM-711B, Kyowa Electronics, Japan). The torque values were calculated as the corresponding tensile forces multiplied by the $0.10 \mathrm{~m}$ lever arm of the force plate.

Each subject was sat back in a dedicated chair and their trunk was secured to the chair using non-elastic straps. Their right foot (dominant side) was secured to the torque-measuring device (Fig. 1). Additionally, 
it was confirmed that the bottom of the toes and foot of the subject did not float from the device in all measurements. After a warming-up session with subjective $60 \%$ contractions, each subject performed the MVC torques of the first and the second-fifth MTPJs at each position for approximately $3 \mathrm{~s}$. The results thus obtained were used to determine the highest torque. The subjects were verbally instructed to avoid counter movement. The MVC torque was calculated as the highest torque minus the lowest torque obtained during contraction (Fig. 2). To avoid the effects of muscle fatigue, the resting period was set as at least $2 \mathrm{~min}$. For analysis, the average torque between the two measurements conducted at each position was chosen. The reliability of the measurements was previously reported [14].

The MVC torques of the first and second-fifth MTPJs were measured at four MTPJ positions and three ankle positions for a total of 12 positions. The MTPJs were measured at $0^{\circ}, 15^{\circ}, 30^{\circ}$, and $45^{\circ}$ dorsiflexed positions $\left(0^{\circ}, \mathrm{DF} 15^{\circ}, \mathrm{DF} 30^{\circ}\right.$, and $\mathrm{DF} 45^{\circ}$, respectively), and the ankle was measured at $20^{\circ}$ plantar-flexed position $\left(\mathrm{PF} 20^{\circ}\right)$, neutral position $\left(0^{\circ}\right)$, and $20^{\circ}$ dorsiflexed position (DF20 $)$. These positions are determined to cover the typical joint angle at push off, and the range of motion during running and sprinting $[15,16]$. The neutral position of the MTPJ was defined as the parallel position between the sole of the foot and toes. The neutral position of the ankle was defined as the perpendicular position between the sole of the foot and longitudinal axis of the fibula. The ankle position was measured using a goniometer prior to each measurement. The MVC torque was measured twice in each position. For both types of MTPJs, the MVC torque values were measured at four MTPJ positions and at a single ankle position per day. This series of measurements was spread over three days for three different ankle positions. The order of the measured MTPJ and ankle was randomly chosen.

\section{Statistical Analysis}

The descriptive data are presented as means \pm SDs. Shapiro-Wilk tests were used to verify a normal distribution, and the results showed that some components did not follow a normal distribution. Therefore, the Friedman test was used for the ankle and MTPJ angles. When significant differences were found in the Friedman test, the Wilcoxon signed-rank test with a Holm correction was used for multiple comparisons. The same method was used to examine the differences in the torque-angle relationships of the first and second-fifth MTPJs. For all the MTPJ and the ankle positions, the MVC torque values were compared between the first and second-fifth MTPJs using the Mann-Whitney U test. Statistical analyses were performed using a statistical software (SPSS Statistics 26 , IBM, USA). For all the tests, the statistical significance was set as $p<0.05$.

\section{Results}

There were significant variations in the ankle positions at $0^{\circ}, \mathrm{DF} 15^{\circ}$, and DF30 of the first MTPJ in the Friedman test ( $p<0.01,<0.01$, and 0.01 , respectively). In contrast, there was no significant difference in the ankle positions at DF $45^{\circ}$ of the first MTPJ $(p=0.15)$. When the first MTPJ was at $0^{\circ}$ to DF $30^{\circ}$, the MVC torques at $\mathrm{PF} 20^{\circ}$ of the ankle were smaller than those at DF20 of the ankle. There were significant variations in the first MTPJ positions at PF20 $0^{\circ}$, and DF20 of the ankle in the Friedman test, $t(p<0.01$, $<0.01$, and 0.01 , respectively). When the ankle was at PF $20^{\circ}$, the MVC torques were significantly different 
from all other torques and increased as the MTPJ dorsiflexed (Fig. 3). When the ankle was at $0^{\circ}$, the MVC torques increased significantly as the MTPJ dorsiflexed in four comparisons $\left(0^{\circ}\right.$ and DF $30^{\circ}, 0^{\circ}$ and DF $45^{\circ}$, $\mathrm{DF} 15^{\circ}$ and $\mathrm{DF} 45^{\circ}$ as well as DF $30^{\circ}$ and $\mathrm{DF} 45^{\circ}$ ). When the ankle was at DF20 $0^{\circ}$, the $\mathrm{MVC}$ torque at $0^{\circ}$ of the MTPJ was significantly lower than that at $\mathrm{DF} 15^{\circ}$ and DF45 $5^{\circ}$. In contrast, there was no significant difference in the $\mathrm{MVC}$ torques at $\mathrm{DF} 15^{\circ}, \mathrm{DF} 30^{\circ}$, and $\mathrm{DF} 45^{\circ}$.

There was no significant variation in the ankle positions at all second-fifth MTPJ positions in the Friedman test ( $p=0.12,0.06,0.06$, and 0.20 , respectively). There were significant differences in the second-fifth MTPJ angles at PF20, $0^{\circ}$, and DF20 of the ankle in the Friedman test $(p<0.01,<0.01$, and 0.03 , respectively). When the ankle was at PF20 $0^{\circ}$, the MVC torques increased significantly as the MTPJ dorsiflexed in four comparisons $\left(0^{\circ}\right.$ and DF $15^{\circ}, 0^{\circ}$ and DF45 $5^{\circ}$ DF $15^{\circ}$ and $\mathrm{DF} 45^{\circ}$ as well as DF30 $0^{\circ}$ and DF45 ${ }^{\circ}$ ) (Fig. 4). When the ankle was at $0^{\circ}$ and DF20 $0^{\circ}$, the MVC torques at $0^{\circ}$ of each MTPJ were significantly lower than those at the other MTPJ angles.

The highest torque values obtained for the first and second-fifth MTPJs, were $11.4 \pm 2.3 \mathrm{~N} \mathrm{~m}$ and $7.8 \pm$ $1.7 \mathrm{~N} \mathrm{~m}$, respectively. The position at which the highest values of torque were obtained was $\mathrm{DF} 45^{\circ}$ of the MTPJ at $0^{\circ}$ of the ankle. According to the Mann-Whitney $U$ test results, the MVC torque of the first MTPJ was found to be larger compared to that of the second-fifth MTPJs at all positions ( $p<0.01$ for all positions).

\section{Discussion}

This study demonstrated the torque-angle relationships for the first and second-fifth MTPJs. The main findings of the present study were 1) the force generation of the first MTPJ was sensitive to the ankle position and 2) the force generation of the first MTPJ was sensitive to the MTPJ angle when the ankle was at $\mathrm{DF0} 0^{\circ}$. To the best of our knowledge, this is the first study to show each force generation characteristic of the first MTPJ and second-fifth MTPJs. To the best of our knowledge, this is the first study to show each force generation characteristic of the first MTPJ and second-fifth MTPJs.

When the first MTPJ was $0^{\circ}$, DF $15^{\circ}$, and DF30 , the MVC torque of the first MTPJ at DF $20^{\circ}$ of the ankle was higher than at PF $20^{\circ}$ of the ankle. However, there was no significant variation in the ankle positions at all second-fifth MTPJ positions. These results supported our hypothesis. The plantar-flexion moment arm of the ankle is larger in the flexor hallucis longus muscle compared to the flexor digitorum longus muscle [8]. As a result, the flexor hallucis longus muscle is varies more in length compared to the flexor digitorum longus muscle during the plantar-flexion/dorsiflexion of the ankle. Therefore, we considered that the extrinsic muscles make a large contribution to the plantar-flexion torque of the MTPJ in the dorsiflexion position of the ankle, and the muscle activity of the first MTPJ becomes relatively large at the dorsiflexed position of the ankle.

The MVC torques of the first MTPJ increased as the MTPJ was dorsiflexed when the ankle was at PF20 and $0^{\circ}$. However, we observed no significant difference between the MVC torques measured at DF $15^{\circ}$ to DF $45^{\circ}$ of the first MTPJ when the ankle was at DF20 ${ }^{\circ}$. The force capacity generated by a muscle fiber is 
altered by the muscle fiber length (force-length relationships) [17]. These results suggested that the ranges of MTPJ and ankle correspond to the ascending arm of the torque-angle relationship at $0^{\circ}$ to DF $45^{\circ}$ of the first MTPJ, when the ankle was at PF20 and $0^{\circ}$, and the plateau region (i.e., optimum angle zone) at $\mathrm{DF} 15^{\circ}$ to $\mathrm{DF} 45^{\circ}$ of the first MTPJ, when the ankle was at DF20 $0^{\circ}$. The maximal torque is generated at DF $20^{\circ}$ of first MTPJ during sprinting [16]. In addition, the ankle lies in the neutral to plantarflexed position when maximal torque is generated at the first MTPJ during sprinting $[15,18]$. The obtained results indicate that plantar-flexion torque of the MTPJs was generated in ascending limb of the torqueangle relationship during sprinting. Therefore, to generate the higher torque, it could be advantageous not to limit the dorsiflexion of the MTPJ during running and sprinting.

The MVC torques of the second-fifth MTPJs increased as the MTPJ was dorsiflexed when the ankle was at PF20 $0^{\circ}$. However, no significant difference could be observed between the MVC torques at DF $15^{\circ}$ to $\mathrm{DF} 45^{\circ}$ of the second-fifth MTPJs when the ankle was at $0^{\circ}$ and DF $20^{\circ}$. The force generation characteristics are different between the first and the second-fifth MTPJs. The torque-angle relationship of the extrinsic and intrinsic muscles was found to be in the optimum angle zone between DF $15^{\circ}$ to DF $45^{\circ}$ when the ankle was at $0^{\circ}$ in the second-fifth MTPJs. In contrast, the torque-angle relationship was in the ascending arm at $0^{\circ}$ in the first MTPJ. A previous study reported that the fifth MTPJ was less dorsiflexed than the first MTPJ in human walking [19]. We considered that the optimum muscle lengths of the second-fifth MTPJs could be shorter than that of the first MTPJ. Therefore, the muscle activity of the first MTPJ becomes relatively large at the dorsiflexed position of the MTPJ.

The highest torques were $11.4 \pm 2.3$ and $7.8 \pm 1.7 \mathrm{~N} \mathrm{~m}$ on the first and second-fifth MTPJs, respectively. The MVC torque of the first MTPJ was larger than second-fifth MTPJ at all positions. The torques measured in the present study were higher than those estimated in a previous study [13], wherein, the productivity of the torque was calculated from the anatomical cross-sectional area and estimated muscle tensions reported in a study of cadavers. However, physiological cross-sectional area has been reported to be more suitable for predicting functional properties than anatomical cross-sectional area [20]. In addition, the force that a muscle can generate per unit area is altered by the number and firing rate of a motor unit [21], and varies from muscle to muscle [22]. Thus, an estimated value may be different from the measured value. Consequently, the in vivo measured torques of this study were higher than the estimated values reported in the previous study. In the present study, the MVC torque was particularly greater in the first MTPJ. During walking, humans push off from an axis between the first and second MTPJ [23]. In such cases, the first MTPJ was greatly dorsiflexed [19]. These walking characteristics possibly contribute to the development of the motor unit of the hallux.

The MVC torque in the present study was lower than the plantar-flexion torque of the MTPJ during running [24]. A previous study reported that the intrinsic foot muscle lengthens and recoils rapidly during the later stance in accordance with the recoil of the foot arch during running [25]. It is considered that this recoil action causes the stretch shortening cycle [26], which results in an increased torque production. 
Some limitations should be noted. The sample size was small, and the subjects were limited to young men and normal structure of their foot in this study. Hence, the applicable range of the results may be limited. Previous studies have shown that arch height is not correlated to toe grip strength [27].

Additionally, the optimal angle for force production is independent of age or gender [28]. However, there is room to investigate the behavior of the torque-angle relationship among the wide population to understand the toe function.

\section{Conclusions}

This study demonstrated the torque-angle relationships for the first and second-fifth MTPJs. When ankle was at $0^{\circ}$, the MVC torque of the first MTPJ increased with the MTPJ dorsiflexion, but the MVC torques of the second-fifth MTPJ were not significantly different at DF $15^{\circ}$ to DF $45^{\circ}$ of the MTPJ when the ankle was at $0^{\circ}$. Furthermore, when the first MTPJ was $0^{\circ}$ to DF $30^{\circ}$, the MVC torque of the first MTPJ at $\mathrm{DF} 20^{\circ}$ of the ankle was higher than at PF $20^{\circ}$ of the ankle. On the other hand, no significant difference existed between the MVC torques of the second-fifth MTPJs at any ankle position. Thus, the present study suggested that the force generation characteristic of the first MTPJ is more sensitive to the MTPJ and ankle position than the second-fifth MTPJs.

\section{Abbreviations}

MTPJ

metatarsophalangeal joint, MVC:maximal voluntary contraction

\section{Declarations}

\section{Ethics approval and consent to participate}

Informed consent was obtained from all participants. This study was approved by the Ethics Committee on Human Research of Waseda University (approval number: 2013 -195).

\section{Consent for publication}

Not applicable.

\section{Availability of data and materials}

The data that support the findings of this study are available from the corresponding author upon reasonable request.

\section{Competing interests}

The authors declare that they have no competing interests. 


\section{Funding}

This work was supported by Grant-in-Aid from the Japan Society for the Promotion of Science Fellows (18J01881).

\section{Authors' contributions}

JS, SI and ST designed the experiments. JS performed the experiments, data analysis, and drafted the manuscript. SI and ST contributed to discussion and review of the manuscript. All authors read and approved the final manuscript prior to the submission.

\section{Acknowledgements}

We would like to thank the participants in the study.

\section{References}

1. Zelik KE, La Scaleia V, Ivanenko YP, Lacquaniti F: Coordination of intrinsic and extrinsic foot muscles during walking. Eur J Appl Physiol 2015, 115:691-701.

2. Kelly LA, Kuitunen S, Racinais S, Cresswell AG: Recruitment of the plantar intrinsic foot muscles with increasing postural demand. Clin Biomech 2012, 27:46-51.

3. Uritani D, Fukumoto T, Matsumoto D, Shima M: The Relationship Between Toe Grip Strength and Dynamic Balance or Functional Mobility Among Community-Dwelling Japanese Older Adults: A Cross-Sectional Study. J Aging Phys Act 2016, 24:459-464.

4. Tsuyuguchi R, Kurose S, Seto T, Takao N, Tagashira S, Tsutsumi H, Otsuki S, Kimura Y: Toe grip strength in middle-aged individuals as a risk factor for falls. J Sports Med Phys Fit 2018, 58:13251330 .

5. Taş S, Ünlüer N, Çetin A: Thickness, cross-sectional area, and stiffness of intrinsic foot muscles affect performance in single-leg stance balance tests in healthy sedentary young females. $J$ Biomech 2020, 99:109530.

6. Williams M, Stutzman L: Strength variation through the range of joint motion. Phys Ther Rev 1959, 39:145-152.

7. Refshauge KM, Chan R, Taylor JL, McCloskey DI: Detection of movements imposed on human hip, knee, ankle and toe joints. $J$ Physiol 1995, 488:231-241.

8. McCullough MB, Ringleb SI, Arai K, Kitaoka HB, Kaufman KR: Moment arms of the ankle throughout the range of motion in three planes. Foot Ankle Int 2011, 32:300-306.

9. Miaki $\mathrm{H}$, Someya $\mathrm{F}$, Tachino $\mathrm{K}$ : A comparison of electrical activity in the triceps surae at maximum isometric contraction with the knee and ankle at various angles. Eur J Appl Physiol Occup Physiol 
1999, 80:185-191.

10. Goldmann JP, Sanno M, Willwacher S, Heinrich K, Bruggemann GP: The potential of toe flexor muscles to enhance performance. J Sports Sci 2013, 31:424-433.

11. Spinner RJ, Howe BM: Leg. In: Gray's Anatomy: the Anatomical Basis of Clinical Practice. Forty-first edn. Edited by Standring S. Amsterdam: Elsevier; 2015: 1400-1417.

12. Saeki J, Nakamura M, Nakao S, Fujita K, Yanase K, Morishita K, Ichihashi N: Ankle and toe muscle strength characteristics in runners with a history of medial tibial stress syndrome. J Foot Ankle Res 2017, 10:16.

13. Green SM, Briggs PJ: Flexion strength of the toes in the normal foot. An evaluation using magnetic resonance imaging. Foot 2013, 23:115-119.

14. Saeki J, Tojima M, Torii S: Relationship between navicular drop and measuring position of maximal plantar flexion torque of the first and second-fifth metatarsophalangeal joints. J Phys Ther Sci 2015, 27:1795-1797.

15. Stefanyshyn DJ, Nigg BM: Dynamic Angular Stiffness of the Ankle Joint during Running and Sprinting. J Appl Biomech 1998, 14:292-299.

16. Smith $G$, Lake M, Lees A: Metatarsophalangeal joint function during sprinting: a comparison of barefoot and sprint spike shod foot conditions. J Appl Biomech 2014, 30:206-212.

17. Gordon AM, Huxley AF, Julian FJ: The variation in isometric tension with sarcomere length in vertebrate muscle fibres. J Physiol 1966, 184:170-192.

18. Stefanyshyn DJ, Nigg BM: Influence of midsole bending stiffness on joint energy and jump height performance. Med Sci Sports Exerc 2000, 32:471-476.

19. Fernandez PJ, Holowka NB, Demes B, Jungers WL: Form and function of the human and chimpanzee forefoot: implications for early hominin bipedalism. Sci Rep 2016, 6:30532.

20. Fukunaga T, Roy RR, Shellock FG, Hodgson JA, Edgerton VR: Specific tension of human plantar flexors and dorsiflexors. J Appl Physiol 1996, 80:158-165.

21. Conwit RA, Stashuk D, Tracy B, McHugh M, Brown WF, Metter EJ: The relationship of motor unit size, firing rate and force. Clin Neurophysiol 1999, 110:1270-1275.

22. Seki K, Narusawa M: Firing rate modulation of human motor units in different muscles during isometric contraction with various forces. Brain Res 1996, 719:1-7.

23. Bojsenmoller F: Calcaneocuboid joint and stability of the longitudinal arch of the foot at high and low gear push off.J Anat 1979, 129:165-176.

24. Farris DJ, Kelly LA, Cresswell AG, Lichtwark GA: The functional importance of human foot muscles for bipedal locomotion. Proc Natl Acad Sci USA 2019, 116:1645-1650.

25. Kelly LA, Lichtwark G, Cresswell AG: Active regulation of longitudinal arch compression and recoil during walking and running. $J R$ Soc Interface 2015, 12:8.

26. Bosco C, Tarkka I, Komi PV: Effect of elastic energy and myoelectrical potentiation of triceps surae during stretch-shortening cycle exercise. Int J Sports Med 1982, 3:137-140. 
27. Uritani $D$, Fukumoto T, Matsumoto $D$, Shima M: Associations between toe grip strength and hallux valgus, toe curl ability, and foot arch height in Japanese adults aged 20 to 79 years: a cross-sectional study. J Foot Ankle Res 2015, 8:18.

28. Winegard KJ, Hicks AL, Vandervoort AA: An evaluation of the length-tension relationship in elderly human plantarflexor muscles. J Gerontol A Biol Sci Med Sci 1997, 52:337-343.

Figures 


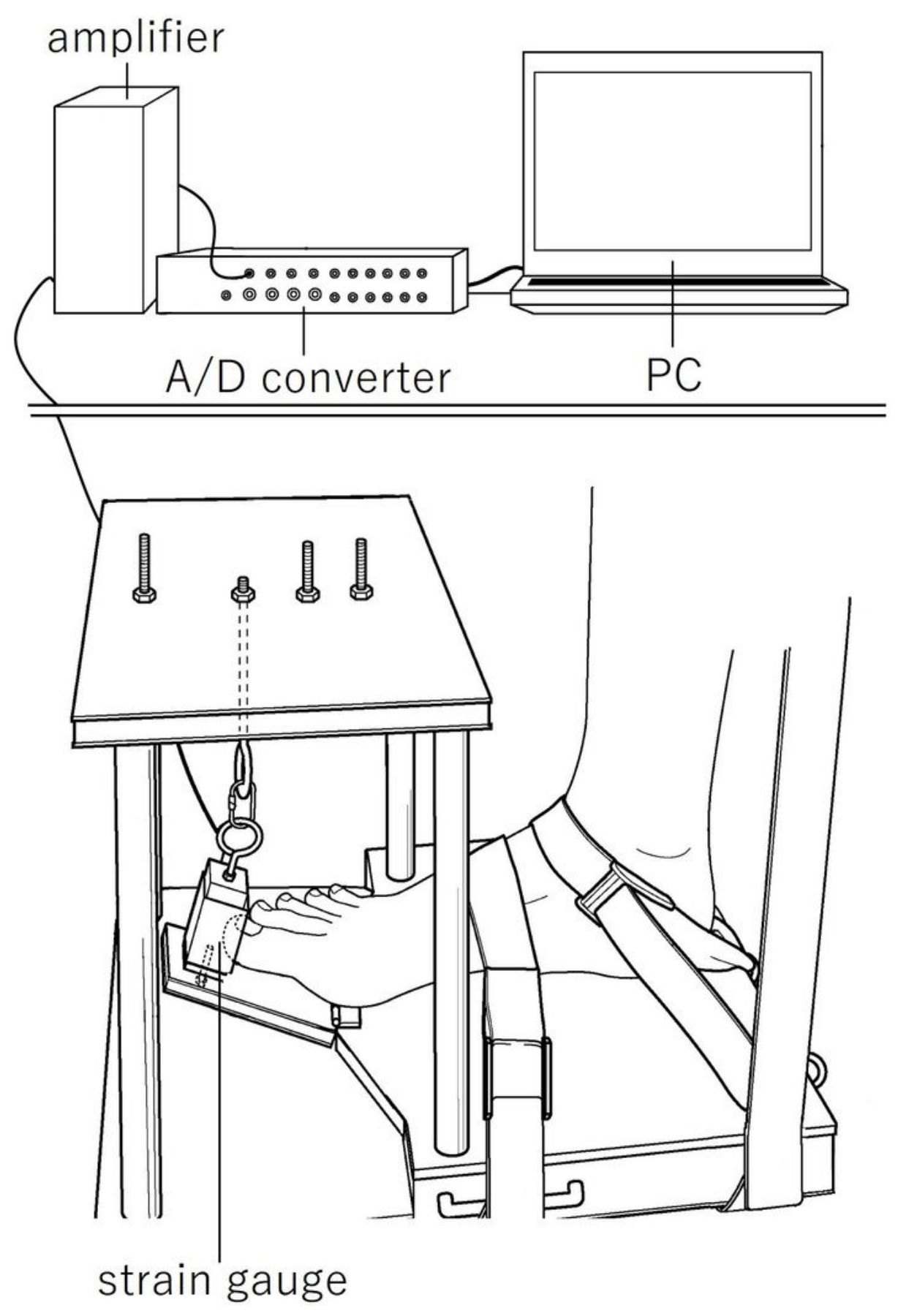

Figure 1

Structure of the metatarsophalangeal joint (MTPJ) plantar-flexion torque-mater for measuring the isometric torque. The plantar-flexion torque was calculated from the tensile force $(\varepsilon)$ and lever arm of the foot plate $(0.10 \mathrm{~m})$. 


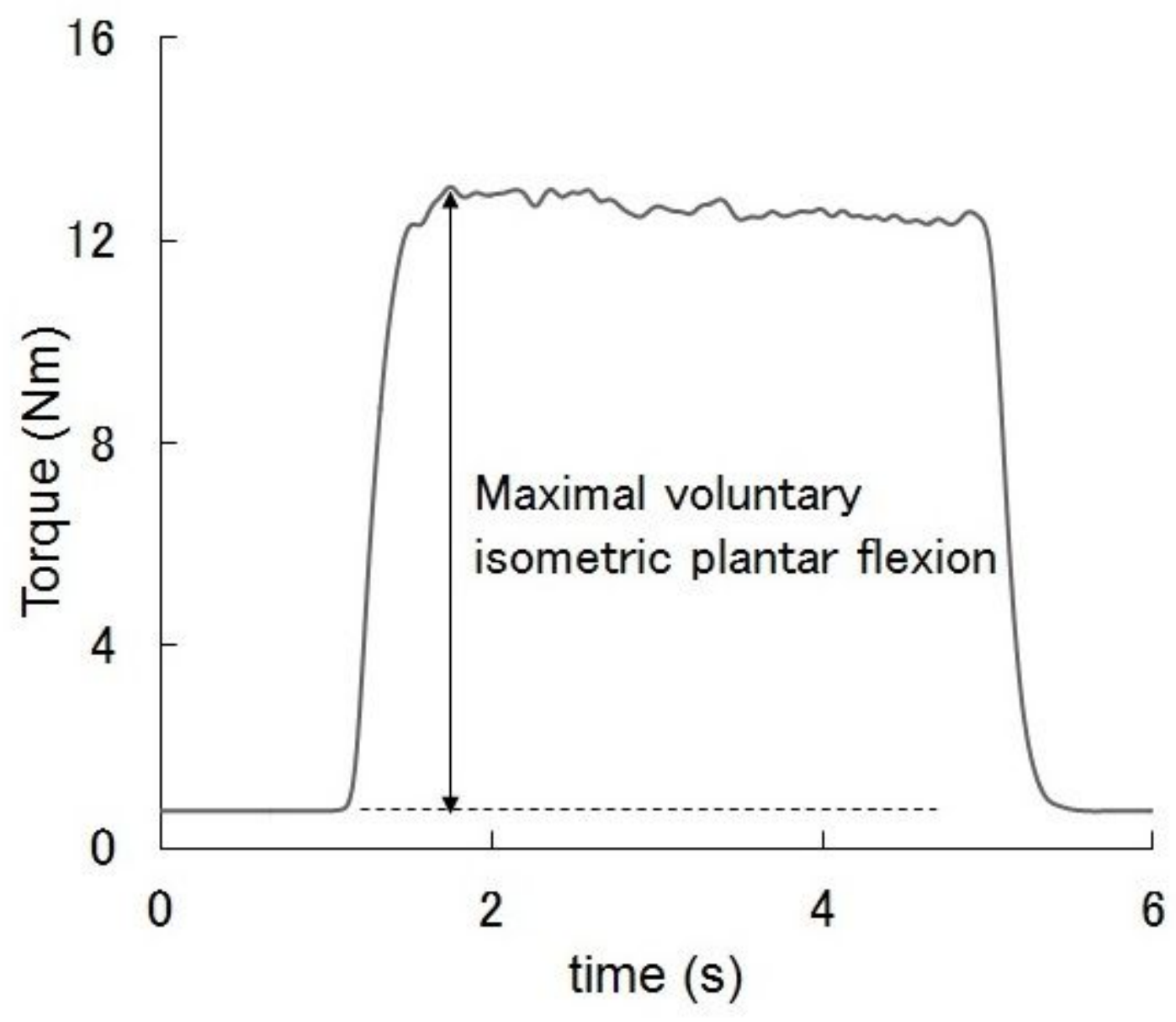

Figure 2

Typical data of the measured maximal voluntary isometric plantar-flexion torque of each metatarsophalangeal joint (MTPJ). Maximal voluntary isometric plantar-flexion torque was defined as the difference between the maximal torque during maximal voluntary isometric contraction (MVC) and passive torque at rest. 

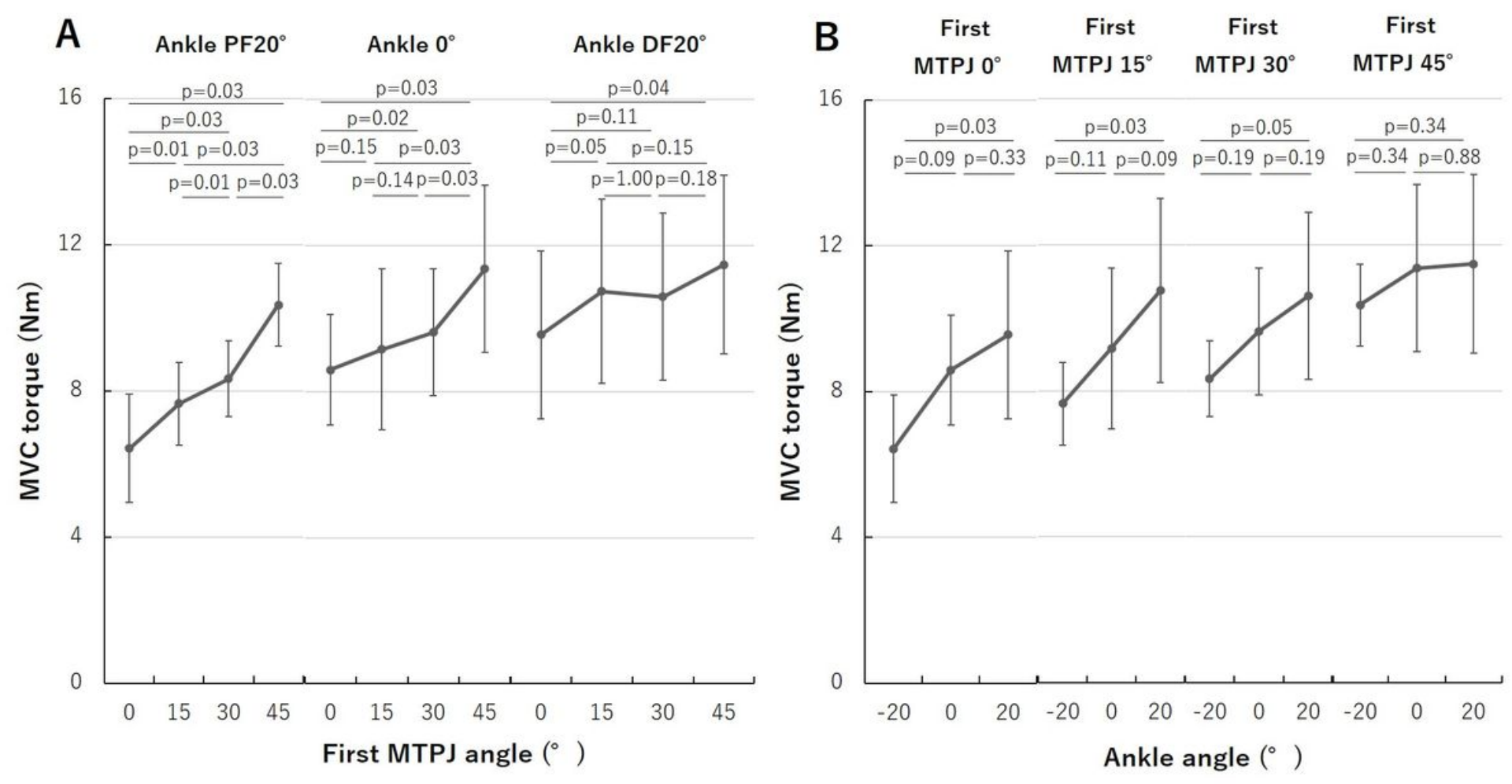

Figure 3

Maximal voluntary isometric contraction (MVC) torque of the first metatarsophalangeal joint (MTPJ) at each ankle position. PF $20^{\circ}: 20^{\circ}$ plantar-flexed position, $0^{\circ}$ : neutral position, DF20 $20^{\circ}$ dorsiflexed position. Numbers indicate the p-value in the multiple comparison test.
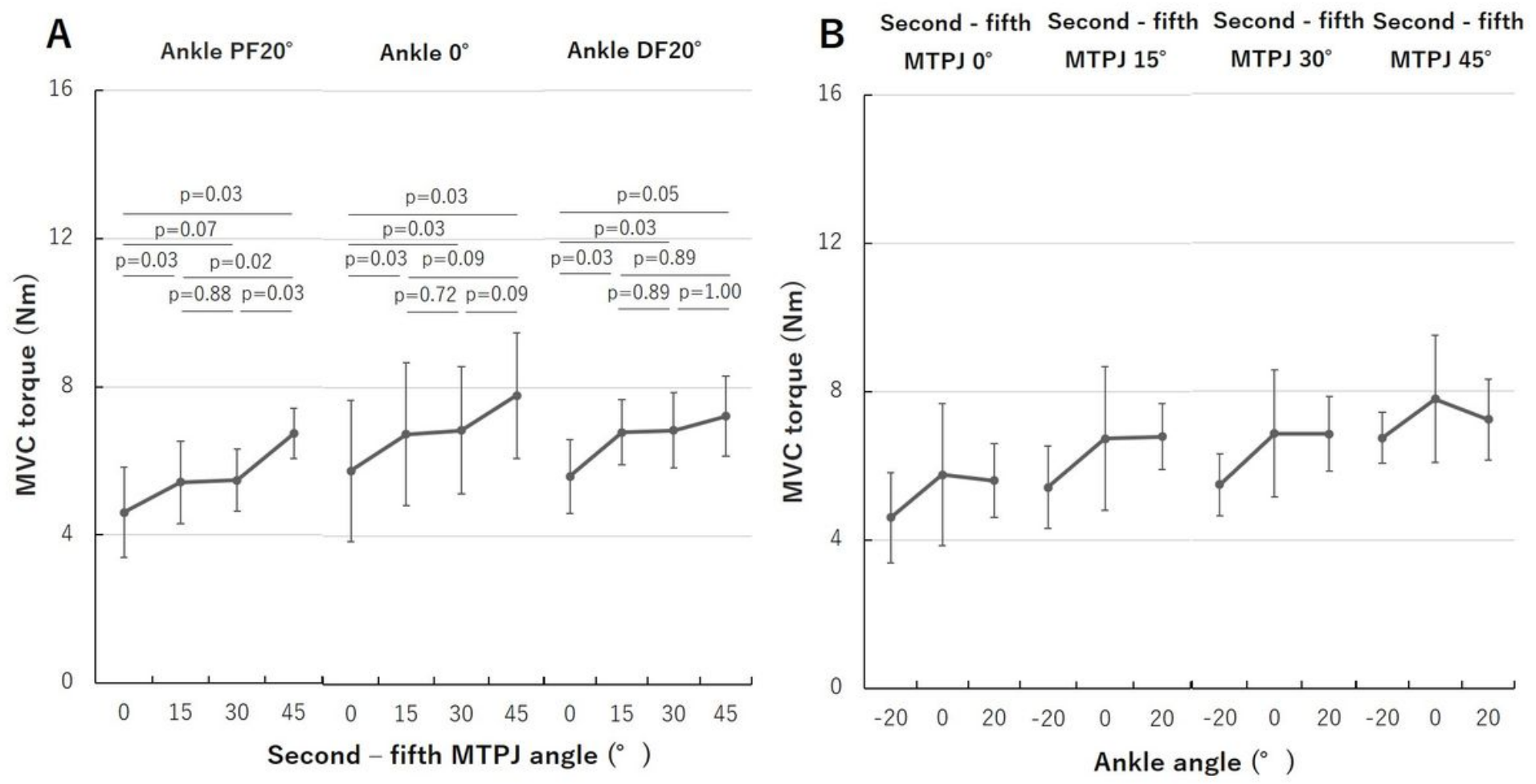


\section{Figure 4}

Maximal voluntary isometric contraction (MVC) torque of the second-fifth metatarsophalangeal joints (MTPJs) at each ankle joint angle. PF20 $20^{\circ}$ plantar-flexed position, $0^{\circ}$ : neutral position, DF20 $20^{\circ}$ dorsiflexed position. Numbers indicate the $\mathrm{p}$-value in the multiple comparison test. 